\title{
On Reducing the Effect of Silhouette Quality on Individual Gait Recognition: a Feature Fusion Approach
}

\author{
Ning Jia, Victor Sanchez, \\ Chang-Tsun Li \\ Dept. of Computer Science \\ University of Warwick \\ Coventry, UK \\ n.jia@warwick.ac.uk \\ vsanchez@dcs.warwick.ac.uk \\ c-t.li@warwick.ac.uk
}

Hassan Mansour

\author{
Mitsubishi Electric \\ Research Labs \\ Massachusetts, US \\ mansour.hassan@gmail.com
}

\begin{abstract}
The quality of the extracted gait silhouettes can hinder the performance and practicability of gait recognition algorithms. In this paper, we propose a framework that integrates a feature fusion approach to improve recognition rate under this situation. Specifically, we first generate a dataset containing gait silhouettes with various qualities based on the CASIA Dataset $B$. We then fuse gallery data with different qualities and project data into embedded subspaces. We perform classification based on the Euclidean distances between fused gallery features and probe features. Experimental results show that the proposed framework can provide important improvements on recognition rate.
\end{abstract}

\section{INTRODUCTION}

As the only biometric source that can be acquired at a distance, gait recognition has drawn great attention over the past decade. Various algorithms have been proposed for individual gait recognition, which can be classified as modelbased or model-free approaches. Model-based approaches rely on the extraction of parameters from the subjects' body and walking cycle to construct a structural model of human motion [1]. Model-free approaches, on the other hand, rely on spatio-temporal representations of gait, which may be directly obtained from the acquired gait sequences. These approaches, in general, provide high recognition accuracy with low computational cost, as they usually use binary silhouette images to represent gait sequences.

Unfortunately, gait is not as reliable as other biometric traits such as fingerprints and iris [2][3]. Factors such as age, clothes, walking surfaces, viewing angles, and health condition may result in poor recognition performance. Furthermore, recognition efficiency may be hindered if the associated gallery and probe gait silhouettes are acquired under different situations. The quality of the gait silhouette can be influenced, for example, by the background environment when capturing gait sequences and the accuracy of the segmentation method used to detect the gait silhouette. A detailed review of gait databases and algorithms can be found in [4].
The effect of the gait silhouette quality on the performance and practicability of model-free gait recognition algorithms is an important issue that has been less intensively studied and only a limited number of solutions are reported in the literature. Sarkar et al. [3] discuss several cases when gait silhouette segmentation errors occur in the HumanID Gait Challenge Problem data set due to the shadow of the individuals, varying lighting conditions and moving objects in the background. In [5], Liu and Sarkar observe that the drop in gait silhouette segmentation quality may lead to a decrease in recognition accuracy. They also observe that if gallery and probe gait sequences are captured under the same conditions, and are segmented by the same method, the recognition accuracy may be high even if the data quality is poor. Zhang et al. [6] address the issue of poor recognition accuracy when low-resolution gait silhouettes are used. The authors propose to combine superresolution with multi-linear tensor-based learning without parameters (SRMTP) to overcome this problem. However, they test their algorithm on artificial dataset, and thus the issue of silhouette quality remains unexplored in practical scenarios. Recently Shaikh et al. [7] propose a partial silhouette-based approach that extracts the hand dynamics as gait signatures, and claim to be efficient on incomplete or distorted silhouettes. However, no result is provided about the performance on lowquality silhouette.

In this work, we consider the situation in which the gait data related to an individual to be recognised (probe data) are not captured under ideal conditions, and therefore the associated gait silhouettes may be noisy and inaccurately segmented; whereas the stored gait data (gallery data) are well segmented, or vice versa. This is a common situation encountered in practice; for example, when the probe data is captured using CCTV cameras at low resolution and poor quality, but the gallery data is previously captured under ideal conditions and it is not feasible to re-capture the probe data under the same ideal conditions. Based on this scenario, we employ various segmentation algorithms to generate different silhouette quality data using sequences from the CASIA 
Dataset B. A subspace learning method is then used to find a low dimension feature subspace. A fusion strategy is employed to fuse gallery data of different quality levels, and the fused gallery data is then matched with the probe data in the feature space. We employ two popular subspace learning methods, namely, Linear Discriminant Analysis (LDA) [8], and Locality Preserving Projection (LPP) [9], to confirm the improvement brought by fusion approach. Experimental results show that the fusion strategy attains high recognition accuracy, making it a promising solution to reduce the negative effects of poor gait silhouette quality on individual recognition.

The rest of the paper is organized as follows. Section 2 briefly reviews the subspace learning methods used in this work. Section 3 details the proposed framework. Section 4 presents the experimental results and related discussions. Finally, Section 5 draws conclusions.

\section{SUBSPACE LEARNING METHODS}

Gait Energy Image (GEI) [2] is an effective gait representation for individual recognition as it reduces computational cost as well as storage space. This work employs such GEIs. Let us assume there are $N$ gait silhouettes, represented as binary images, in one gait period. A GEI $G(x, y)$ is defined as $G(x, y)=\frac{1}{N} \sum_{k=1}^{N} I_{k}(x, y)$, where $I_{k}(x, y)$ is the $k$ th binary image, and $(x, y)$ denotes the pixel coordinates. Examples of GEIs are shown at the rightmost column of Fig. 2.

Consider $n$ GEI samples that are stored as $d$-dimensional column vectors in a matrix $\mathbf{X}=\left\{\mathbf{x}_{\mathbf{1}}, \ldots, \mathbf{x}_{\mathbf{n}}\right\}, \mathbf{x}_{\mathbf{i}} \in \mathfrak{R}^{d}, i \in$ $\{1,2, \ldots, n\}$. Let $W$ be the transformation matrix that projects the original space onto an $r$-dimensional subspace, where $d \gg$ $r$. The new feature matrix in the subspace is denoted as $\mathbf{Y}=$ $\left\{\mathbf{y}_{\mathbf{1}}, \ldots, \mathbf{y}_{\mathbf{n}}\right\}$, where $y_{i} \in \mathfrak{R}^{r}$. The transformation matrix for each element is given by $\mathbf{y}_{\mathbf{i}}=W^{T} \mathbf{x}_{\mathbf{i}}, i \in\{1, \ldots, n\}$. Matrix $W$ varies according to the subspace learning method used.

\section{A. Dimentionality Reduction: PCA}

PCA is used as an approach to avoid singularities in further covariance matrix calculations [8], for example in LDA and LPP. PCA seeks a compact representation of patterns in a feature subspace. The columns of the PCA transformation matrix $W_{P C A}$ are calculated by solving the eigendecomposition problem $\lambda_{i} \mathbf{e}_{\mathbf{i}}=S \mathbf{e}_{\mathbf{i}}$, where $\lambda_{i}$ and $\mathbf{e}_{\mathbf{i}}$ are the corresponding eigenvalues and eigenvectors, respectively, and $S=\frac{1}{n} \sum_{i=1}^{n}\left(\mathbf{x}_{\mathbf{i}}-\mu\right)\left(\mathbf{x}_{\mathbf{i}}-\mu\right)^{\top}$ is the covariance matrix of the original sample matrix $X$, where $\mu$ is the sample mean, $\mu=\frac{1}{n} \sum \mathbf{x}_{\mathbf{i}}, i \in\{1,2, \ldots, n\}$. Matrix $W_{P C A}$ is then composed by column eigenvectors corresponding the $r$ th highest eigenvalues; $W_{P C A}=\left\{\mathbf{e}_{\mathbf{1}}{ }^{\prime}, \mathbf{e}_{\mathbf{2}}{ }^{\prime}, \ldots, \mathbf{e}_{\mathbf{r}}{ }^{\prime}\right\}$, where $\mathbf{e}_{\mathbf{j}}{ }^{\prime}, j \in[1, r]$ is the $j$ th eigenvector.

\section{B. Discriminant Analysis: LDA}

Compared to PCA, LDA embeds discriminant power between different classes in the feature subspace, which makes it a supervised subspace learning method suitable for multi-class learning problems. Assuming there are $c$ classes in $X$, with $n_{l}$ samples in subset $\mathcal{X}_{l}, l \in\{1,2, \ldots, c\}$, so that $n=\sum_{l=1}^{c} n_{l}$; the within-class scatter matrix $S_{W}$ is then defined as: $S_{W}=$ $\sum_{l=1}^{c} \sum_{\mathbf{x} \in \mathcal{X}_{l}}\left(\mathbf{x}-\mu_{\mathbf{l}}\right)\left(\mathbf{x}-\mu_{\mathbf{l}}\right)^{\top}$, and the between-class scatter matrix $S_{B}$ is defined as: $S_{B}=\sum_{l=1}^{c}\left(\mu_{\mathbf{l}}-\mu\right)\left(\mu_{\mathbf{l}}-\mu\right)^{\top}$, where $\mu_{\mathrm{l}}$ is the mean of the samples in class $l$, and $\mu$ is the mean of all samples. In order to maximize between-class scatter while minimizing within-class scatter after projection, the following criterion is used:

$$
W_{L D A}=\underset{W}{\arg \max } \frac{\left|W^{\top} S_{B} W\right|}{\left|W^{\top} S_{W} W\right|}
$$

where $W_{L D A}$ is the transformation matrix, whose columns are the generalised eigenvectors $\left\{\mathbf{e}_{\mathbf{1}}, \mathbf{e}_{\mathbf{2}}, \ldots, \mathbf{e}_{\mathbf{r}}\right\}$ that correspond to the largest eigenvalues in $S_{B} W_{L D A}=\lambda_{i} S_{W} W_{L D A}$.

\section{Manifold Learning Method: LPP}

LPP tends to preserve the local data structure after projecting the data onto a subspace [10]. It first constructs an adjacency graph $G$ to model the local structure of the samples. The adjacency graph has $n$ nodes, with node $i$ corresponding to $\mathbf{x}_{\mathbf{i}}$ in $\mathbf{X}$. A pair of nodes $i$ and $j$ are connected if $\mathbf{x}_{\mathbf{i}}$ and $\mathbf{x}_{\mathbf{j}}$ are close in the space. The elements of the weighted similarity matrix $A$, which specifies the similarities among nodes in $G$, are formulated as follows:

$$
A_{i j}= \begin{cases}\exp \left(\frac{-\left\|\mathbf{x}_{\mathbf{i}}-\mathbf{x}_{\mathbf{j}}\right\|^{2}}{t}\right), & \text { if nodes } i \text { and } j \text { are connected, } \\ 0, & \text { otherwise. }\end{cases}
$$

The heat kernel parameter $t \in \mathfrak{R}$ can be determined empirically; if $t$ is very large, $\exp \left(-\left\|\mathbf{x}_{\mathbf{i}}-\mathbf{x}_{\mathbf{j}}\right\|^{2} / t\right)=1$ and matrix $A$ comprises binary weights. Two possible ways exist to determine if nodes are close:

1. $K$ nearest neighbours: if $\mathbf{x}_{\mathbf{i}}$ is among the $K$ nearest neighbours of $\mathbf{x}_{\mathbf{j}}$, or vice versa;

2. $\epsilon$-nearest neighbours: if $\left\|\mathbf{x}_{\mathbf{i}}-\mathbf{x}_{\mathbf{j}}\right\|^{2}<\epsilon, \epsilon \in \Re$.

The eigen-decomposition problem of LPP is generalized as

$$
X L X^{\top} W_{L P P}=\lambda X D X^{\top} W_{L P P},
$$

where $D$ is a diagonal matrix with $D_{i i}=\sum_{j} A_{i j}$, and $L$ is the Laplacian matrix $L=D-A$. The Laplacian of the graph is an approximation of the Laplace-Beltrami operator. The transformation matrix $W_{L P P}=\left\{\mathbf{e}_{\mathbf{1}}, \mathbf{e}_{\mathbf{2}}, \ldots, \mathbf{e}_{\mathbf{r}}\right\}$, and $\left\{\lambda_{1}, \lambda_{2}, \ldots, \lambda_{r}\right\}$ are the corresponding $r$ smallest eigenvalues. The feature subspace created by $W_{L P P}$ can preserve an intrinsic geometric structure of the manifold samples [10][11][12]. LPP can perform supervised learning by assigning a weight equal to 0 to all between-class similarity matrix values. The total similarity matrix $A$ is then given as follows:

$$
A=\left[\begin{array}{ccc}
A_{1} & \cdots & 0 \\
\vdots & \ddots & 0 \\
0 & 0 & A_{c}
\end{array}\right]
$$

In our experiments, we employ supervised LPP.

\section{PROPOSED FRAMEWORK}

The block diagram of the proposed framework is shown in Fig. 1. We first generate the GEIs with different qualities for the training, gallery and probe data. Using the training data, we compute the transformation matrix corresponding to the subspace learning method. We fuse gallery data with a set of weights computed by least square fitting. Fused gallery data and probe data are transformed into fused gallery features and probe features in a lower dimension space. Finally, we measure the similarities between fused gallery features and probe features. 


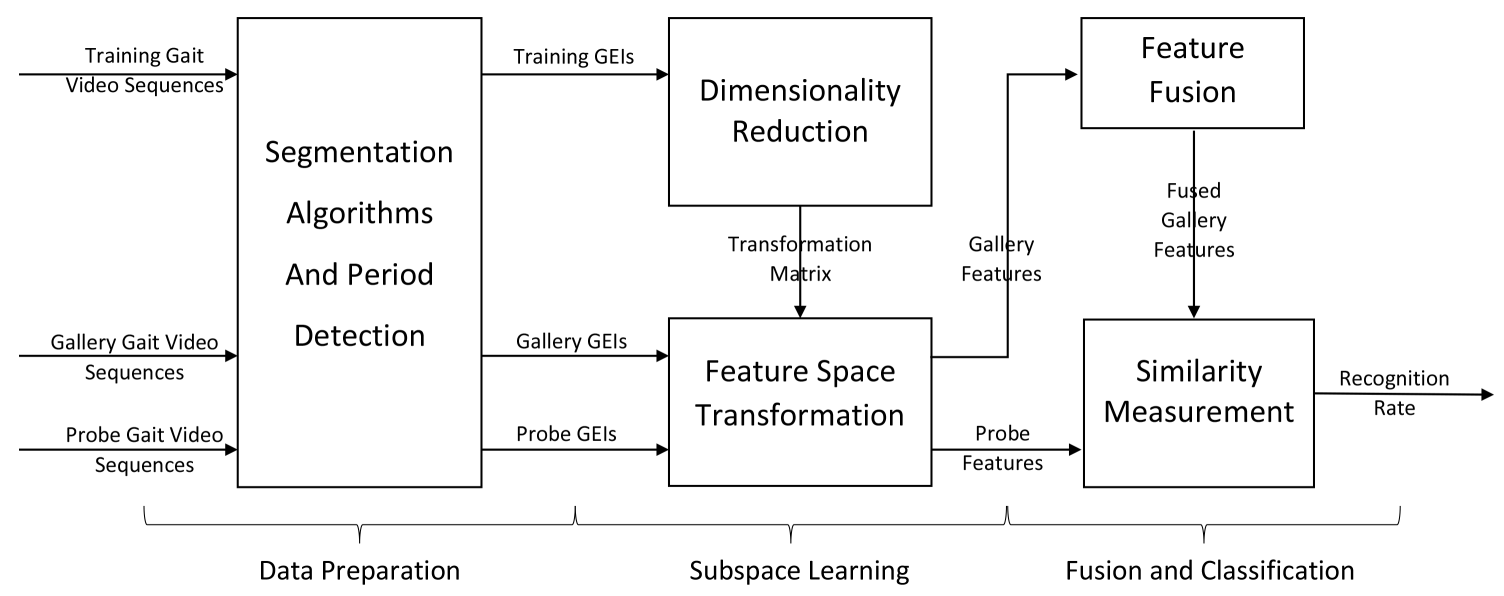

Fig. 1. Block diagram of the proposed framework.

TABLE I. GAIT SILHOUETTE QUALITY LEVELS AND THE CORRESPONDING NOTATIONS

\begin{tabular}{|c|l|}
\hline Quality & \multicolumn{1}{|c|}{ Segmentation approach used } \\
\hline Q.1 & Approach 1: BS method using Otsu's threshold \\
\hline Q.2 & $\begin{array}{l}\text { Approach 2: Normalised BS method plus dilation \& } \\
\text { erosion }\end{array}$ \\
\hline Q.3 & Approach 3: BS method with small threshold \\
\hline Q.4 & $\begin{array}{l}\text { Approach 4: Frame differentiation plus dilation \& } \\
\text { erosion }\end{array}$ \\
\hline Q.5 & Approach 5: GMM \& EM method \\
\hline Q.6 & Approach 6: LMedS method \\
\hline
\end{tabular}

\section{A. Segmentation algorithms}

In order to create gait silhouettes of different qualities, we combine different background subtraction (BS) methods with de-noising methods to create four different silhouette segmentation approaches. We also employ the Gaussian Mixture Model and Expectation Maximization(GMM and EM) method [3], and Least Median of Squares (LMedS) method [13], as additional segmentation approaches. The segmented silhouettes obtained by each of these approaches is used to generate binary images (and GEIs) at a specific quality. The quality levels and the corresponding segmentation approaches used are listed in Table I. The segmentation approaches are explained in the following paragraphs.

Approach 1: A pixel is marked as foreground if $\left|I_{t}-B_{t}\right|>$ threshold, where $I_{t}$ refers to an image with both foreground and background objects and $B_{t}$ contains only background objects. The threshold is set using Otsu's method[14].

Approach 2: The background image is normalized to eliminate the negative effects of noise, i.e. $\left|I_{t}-\operatorname{avg} B_{t}\right|>$ threshold, where $\operatorname{avg} B_{t}=B_{t} / \sum p_{i, j}, p_{i, j}$ refers to the value of pixel $i, j$ in $B_{t}$. The threshold is set using Otsu's method. As the obtained foreground may comprise several disconnected regions, dilation and erosion operations are performed to generate the final foreground.

Approach 3: A small threshold is used in order to introduce a distinct contrast in the segmented silhouettes and to include more background objects in the foreground; namely $\left|I_{t}-B_{t}\right|>$ threshold $/ 3$. The threshold is set using Otsu's method.

Approach 4: Frame differentiation is used to mark the moving foreground pixels, $I_{t}-I_{t-1}>$ threshold, where the threshold

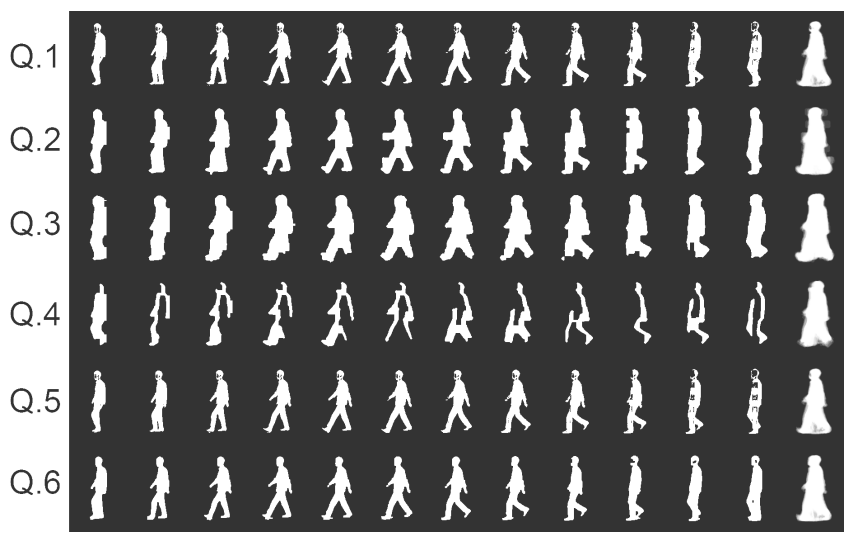

Fig. 2. Sample gait silhouette binary images and their corresponding GEIs (right -most column) computed at six different qualities for the same subject.

is set using Otsu's method. In addition, dilation and erosion operations are used in order to connect the disconnected regions comprising the foreground.

Approach 5: The GMM and EM method, as introduced in the baseline algorithm of Sarkar et al. [3].

Approach 6: The LMedS method, as is introduced in [13], and provided in the CASIA B dataset.

Six silhouette qualities are generated for each gait sequence, including the original silhouette provided by CASIA B dataset denoted as Q.6, as summarized in Table I. This different qualities allow representing differences between the quality of the probe and gallery data. For each quality, we compute the corresponding GEIs following the work of Han et al. [2]. Fig. 2 shows visual samples of silhouettes and corresponding GEIs computed for the different qualities.

\section{B. Feature extraction}

Different subspace learning methods may be used to project the data onto a feature subspace. In this work, to avoid singularity problems in computation, we employ PCA before implementing LDA, or LPP. The generated transformation matrix $W_{\text {trans }}$ is then $W_{\text {trans }}=W_{s}^{\top} W_{P C A}^{\top}$, where $s \in$ $\{L D A, L P P\}$. 
TABLE II. MATCHING RATES BETWEEN GALLERY DATA (G) AND PROBE DATA (P) WITHOUT DIMENSIONALITY REDUCTION AND SUBSPACE LEARNING. (\%)

\begin{tabular}{|c|c|c|c|c|c|c|}
\hline G P & Q.1 & Q.2 & Q.3 & Q.4 & Q.5 & Q.6 \\
\hline Q.1 & $\mathbf{8 5}$ & 12 & 7 & 10 & 80 & 70 \\
\hline Q.2 & 12 & $\mathbf{6 7}$ & 17 & 8 & 10 & 35 \\
\hline Q.3 & 17 & 15 & $\mathbf{7 8}$ & 5 & 17 & 8 \\
\hline Q.4 & 15 & 8 & 5 & $\mathbf{3 8}$ & 18 & 15 \\
\hline Q.5 & 83 & 12 & 7 & 13 & $\mathbf{8 3}$ & 63 \\
\hline Q.6 & 58 & 25 & 5 & 10 & 43 & $\mathbf{9 7}$ \\
\hline
\end{tabular}

\section{Similarity computation}

The feature sets after projection are:

$$
\begin{aligned}
& \{\hat{\mathbf{g}}\}: \hat{g}_{i}=W_{\text {trans }} G_{i} \\
& \{\hat{\mathbf{p}}\}: \hat{p}_{j}=W_{\text {trans }} P_{j}
\end{aligned}
$$

where $i=\left\{1,2, \ldots, n_{1}\right\}, j=\left\{1,2, \ldots, n_{2}\right\}$, and $n_{1}, n_{2}$ are the total number of GEIs in gallery and probe data sets, respectively. The centroid of class $l$ in $\{\hat{\mathbf{g}}\}$ is calculated as $\mathbf{m g}_{\mathbf{1}}=\frac{1}{n_{l}} \sum_{\hat{\mathbf{g}} \in \hat{\mathbf{g}}_{l}} \hat{g}_{i}$, where $\hat{\mathbf{g}}_{l}$ is the set of gallery feature vectors in class $l$. The centroid of class $l$ in $\{\hat{\mathbf{p}}\}$ is calculated in the same way and is denoted as $m p_{l}$. The classifier is then defined as:

$$
D\left(\mathbf{m g}_{\mathbf{l}}, \mathbf{m p}_{\mathbf{i}}\right)=\left\|\mathbf{m p}_{\mathbf{i}}-\mathbf{m g}_{\mathbf{l}}\right\|, i=1,2, \ldots c .
$$

If $D\left(\mathbf{m g}_{\mathbf{l}}, \mathbf{m p}_{\mathbf{l}}\right)=\min _{i=1}^{c} D\left(\mathbf{m g}_{\mathbf{l}}, \mathbf{m p}_{\mathbf{i}}\right)$, the probe feature vector is assigned to the right class label.

\section{EXPERIMENTAL EVALUATION}

In order to evaluate the framework, we use the gait sequences of CASIA B dataset to generate the GEIs at different qualities. CASIA B dataset comprises video sequences for 124 individuals. The frame size is $320 \times 240$, and the frame rate is $25 \mathrm{fps}$. As this work aims at studying the effect of gait silhouette quality on recognition, other factors that may influence the recognition performance are excluded. Therefore, only normal gait sequences are chosen form CASIA B, without the factors of carrying bags, different clothes, different view angles, etc.

\section{A. Evaluation without subspace learning}

We first evaluate the performance with no dimensionality reduction or subspace learning method. The matching rates in percentage are tabulated in Table 2. Two observations can be drawn from the table:

1. The entries in the main diagonal represent the matching results between gallery and probe data of the same quality. These values are usually the highest values in each row which shows that when both gallery and probe data have the same quality, even if the quality is poor, the best matching results are attained. In our experiment, it is shown that silhouettes of quality Q.1 and Q.5 have smaller distances (i.e., the matching rate between them is relatively high), which means the quality is similar, even though the segmentation algorithms are totally different.

2. The entries outside the main diagonal show that the discrepancy in gait silhouette quality between gallery and probe data indeed decreases the recognition accuracy. In some cases, the matching rate between data segmented using the same algorithm can also be very low, which indicates that the segmentation algorithm may be inappropriate for the video source (see for example Q.4 matched with Q.4).

\section{B. Evaluation with subspace learning}

Tables III and IV tabulate the average matching rates in percentage, after using PCA+LDA and PCA+LPP respectively. It is important to recall that in practical cases, the quality of the gallery and probe data may differ. It is then important for recognition algorithms to maintain a high accuracy even in this situation. Therefore, we measure the similarity between each individual in the probe data set against all individuals in the gallery data set for all qualities except for the quality of the probe data. This scenario corresponds to the empty entries in Table III and Table IV. These two tables show that by using dimensionality reduction plus a subspace learning method, matching rates can be considerably improved. Note that LPP can effectively deal with poor quality matching, i.e., qualities different from Q.6, while LDA appears to perform better than LPP with high quality matching, i.e., Q.6 data.

\section{Evaluation with fusion strategy}

We propose to further improve the performance of subspace learning methods by fusing gallery data before matching with probe data features. Specially, we fuse the multi GEI representation of the gallery subjects, which consists of various quality levels, into one GEI representation.

Before feature space transformation, we compute a set of weights to be used in fusion strategy. In this experiment, for each probe data of a specific quality, there are gallery data of 5 different qualities available for fusion. For example, for probe data of quality Q.1, we fuse gallery data of all qualities except quality Q.1. The set of weights for fusion are computed using least squares fitting. Specifically, we aim to find the combination of weights for the gallery data centroids, i.e., gallery data of different qualities, that best match the centroid of the probe data of a specific quality. Let us denote the set of GEI vectors in the gallery set as $\mathbf{G}=\left\{\mathbf{g}_{1}, \mathbf{g}_{2}, \ldots, \mathbf{g}_{\mathbf{n}}\right\}$, for $n$ different qualities. Let us also denote the probe GEI vector as $\mathbf{p}$. The set of weights $\mathbf{w}=\left\{w_{1}, w_{2}, \ldots, w_{n}\right\}$ for the gallery data of $n$ different qualities is then computed as follows:

$$
\mathbf{w}=\underset{\mathbf{w}}{\arg \min }\left\|\mathbf{G} * \mathbf{w}^{\top}-\mathbf{p}\right\|
$$

These weights are then used to fuse gallery data into $\mathbf{G}_{\mathbf{f}}$ :

$$
\mathbf{G}_{\mathbf{f}}=\sum_{i}^{n} \mathbf{g}_{\mathbf{i}} * w_{i}, i \in\{1,2, \ldots, n\}
$$

After fusion, the gallery data is projected into the feature space, where similarities are measured between probe and the fused gallery features. The results of this experiment are shown in Table V. In this table, for comparison, we average the column rates of Table III and IV, and denote them as 'LDA' and 'LPP' respectively. 
TABLE III. MATChing RATES BETWEen GALLERY DATA (G) AND PROBE DATA (P) USING PCA+LDA (\%)

\begin{tabular}{|c|c|c|c|c|c|c|}
\hline G P & Q.1 & Q.2 & Q.3 & Q.4 & Q.5 & Q.6 \\
\hline Q.1 & & 73.3 & 63.3 & 28.3 & 93.3 & 95 \\
\hline Q.2 & 85 & & 86.7 & 31.7 & 75 & 90 \\
\hline Q.3 & 71.7 & 73.3 & & 28.3 & 70 & 83.3 \\
\hline Q.4 & 61.7 & 51.7 & 71.7 & & 58.3 & 70 \\
\hline Q.5 & 95 & 73.3 & 63.3 & 23.3 & & 96.7 \\
\hline Q.6 & 85 & 70 & 66.7 & 28.3 & 85 & \\
\hline
\end{tabular}

TABLE IV. MATCHING RATES BETWEEN GALLERY DATA (G) AND PROBE DATA (P) USING PCA+LPP (\%)

\begin{tabular}{|c|c|c|c|c|c|c|}
\hline G P & Q.1 & Q.2 & Q.3 & Q.4 & Q.5 & Q.6 \\
\hline Q.1 & & 71.7 & 75 & 28.3 & 96.7 & 93.3 \\
\hline Q.2 & 83.3 & & 85 & 35 & 83.3 & 93.3 \\
\hline Q.3 & 78.3 & 73.3 & & 26.7 & 75 & 83.3 \\
\hline Q.4 & 66.7 & 53.3 & 63.3 & & 66.7 & 65 \\
\hline Q.5 & 96.7 & 68.3 & 68.3 & 26.7 & & 93.3 \\
\hline Q.6 & 88.3 & 65 & 66.7 & 20 & 91.7 & \\
\hline
\end{tabular}

\section{Discussions}

In cases when the data quality in the gallery set is different from that of the data in the probe set, the performance of recognition algorithms may be poor, making it hard to chose a dependable classifier. The fusion strategy proposed in this work finds the combination of gallery data that has a minimum distance to the probe data. This is done by finding a set of weights using least square fitting, which is efficient and parameter-free. In our experiment design, we assume that the silhouette quality of probe data does not match any of the quality in gallery data, which would be more frequently occurred for practical cases. As show in Table V, this strategy can considerably improve recognition performance under such circumstances. It is important to mention that by introducing least square fitting, the multi-quality gallery data is fused to best fit the probe data, which is similar to the case where gallery and probe data are equally segmented, i.e. the diagonal data in Table II.

\section{CONCLUSION}

This paper presented a framework for GEI-based gait recognition for cases when a discrepancy in quality between gallery and probe data exists. The motivation is to tackle the problem where gallery and probe data are segmented using different algorithms. To this end, we generate GEIs with different qualities in order to represent segmentation inaccuracies commonly encountered when dealing with low quality data. To perform recognition, we study the use of subspace learning methods after dimensionality reduction by PCA. Simulation experiments on the CASIA B dataset using LDA and LPP

TABLE V. AVERAGE MATCHING RATE OF SIX DIFFERENT QUALITY GALLERY DATA (G) AND PROBE DATA (P) USING PCA+LDA (LDA) AND PCA+LPP (LPP), AND THE MATCHING RATE OF FUSED GALLERY DATA USING PCA+LDA WITH FEATURE FUSION (LDAF) AND PCA+LPP WITH FEATURE FUSION (LPPF)(\%)

\begin{tabular}{|c|c|c|c|c|c|c|c|}
\hline G P & Q.1 & Q.2 & Q.3 & Q.4 & Q.5 & Q.6 & Average \\
\hline LDA & 81 & 72 & 74 & 32 & 79 & 88 & $\mathbf{6 8 . 2}$ \\
\hline LDAF & 75 & 78.3 & 93.3 & 35 & 93.3 & 95 & $\mathbf{7 8 . 3}$ \\
\hline LPP & 84 & 72 & 75 & 31 & 85 & 90 & $\mathbf{6 9 . 4}$ \\
\hline LPPF & 78.3 & 75 & 90 & 31.7 & 95 & 95 & $\mathbf{7 7 . 5}$ \\
\hline
\end{tabular}

indicate that gait recognition is indeed affected if the quality of the probe data set differs from that of the gallery data set. Results also suggest that important improvements in matching rate may be attained when subspace learning methods are used, since the feature subspace finds the best projection to match probe with gallery features of the same quality level. The paper also presented a fusion strategy that fuses gallery data of different qualities before feature space transformation. Experiments showed that this fusion strategy, which employs a number of weights, can further improve matching rates.

\section{REFERENCES}

[1] C. Wang, J. Zhang, L. Wang, J. Pu, and X. Yuan, "Human identification using temporal information preserving gait template," Pattern Analysis and Machine Intelligence, IEEE Transactions on, vol. 34, no. 11, pp. 2164-2176, Nov 2012.

[2] J. Han and B. Bhanu, "Individual recognition using gait energy image," Pattern Analysis and Machine Intelligence, IEEE Transactions on, vol. 28, no. 2, pp. 316-322, 2006.

[3] S. Sarkar, P. Phillips, Z. Liu, I. Vega, P. Grother, and K. Bowyer, "The humanid gait challenge problem: data sets, performance, and analysis," Pattern Analysis and Machine Intelligence, IEEE Transactions on, vol. 27, no. 2, pp. 162-177, Feb 2005.

[4] Y. Makihara, D. Matovski, M. S. Nixon, J. N. Carter, and Y. Yagi, Gait Recognition: Databases, Representations, and Applications. Wiley, 2015.

[5] Z. Liu and S. Sarkar, "Effect of silhouette quality on hard problems in gait recognition," Systems, Man, and Cybernetics, Part B: Cybernetics, IEEE Transactions on, vol. 35, no. 2, pp. 170-183, April 2005.

[6] J. Zhang, J. Pu, C. Chen, and R. Fleischer, "Low-resolution gait recognition," Systems, Man, and Cybernetics, Part B: Cybernetics, IEEE Transactions on, vol. 40, no. 4, pp. 986-996, Aug 2010.

[7] S. Shaikh, K. Saeed, and N. Chaki, "Gait recognition using partial silhouette-based approach," pp. 101-106, Feb 2014.

[8] A. Webb and K. Copsey, Statistical Pattern Recognition, 3rd ed. Chichester: Wiley, 2011.

[9] P. Belhumeur, J. Hespanha, and D. Kriegman, "Eigenfaces vs. fisherfaces: recognition using class specific linear projection," Pattern Analysis and Machine Intelligence, IEEE Transactions on, vol. 19, no. 7, pp. 711-720, Jul 1997.

[10] X. He and P. Niyogi, "Locality preserving projections," Advances in Neural Information Processing Systems(NIPS), vol. 16, pp. 234-241, 2003.

[11] X. He, S. Yan, Y. Hu, P. Niyogi, and H.-J. Zhang, "Face recognition using laplacianfaces," Pattern Analysis and Machine Intelligence, IEEE Transactions on, vol. 27, no. 3, pp. 328-340, March 2005.

[12] M. Belkin and P. Niyogi, "Laplacian eigenmaps for dimensionality reduction and data representation," Neural computation, vol. 15, no. 6, pp. 1373-1396, 2003.

[13] L. Wang, T. Tan, H. Ning, and W. Hu, "Silhouette analysis-based gait recognition for human identification," Pattern Analysis and Machine Intelligence, IEEE Transactions on, vol. 25, no. 12, pp. 1505-1518, Dec 2003.

[14] M. Sezgin and B. Sankur, "Survey over image thresholding techniques and quantitative performance evaluation," Journal of Electronic Imaging, vol. 13, no. 1, pp. 146-168, 2004. [Online]. Available: http://dx.doi.org/10.1117/1.1631315 